\title{
Most Plasmodiophora brassicae Populations in Single Canola Root Galls from Alberta Fields are Mixtures of Multiple Strains
}

\author{
Heting Fu, ${ }^{1}$ Yalong Yang, ${ }^{1}$ Vachaspati Mishra, ${ }^{1}$ Qixing Zhou, ${ }^{1}$ Krista Zuzak, ${ }^{1}$ David Feindel, ${ }^{2}$ Michael W. Harding, ${ }^{3, \dagger}$ and Jie Feng ${ }^{1, \dagger}$ \\ ${ }^{1}$ Alberta Plant Health Lab, Crop Diversification Centre North, Alberta Agriculture and Forestry, Edmonton, AB, T5Y 6H3, \\ Canada \\ ${ }^{2}$ Crop Diversification Centre North, Alberta Agriculture and Forestry, Edmonton, AB, T5Y 6H3, Canada \\ ${ }^{3}$ Crop Diversification Centre South, Alberta Agriculture and Forestry, Brooks, AB, T1R 1E6, Canada
}

\begin{abstract}
Clubroot, caused by Plasmodiophora brassicae, is an important disease on canola in Alberta, Canada. The pathogen is grouped into pathotypes according to their virulence reaction on differential hosts. Multiple pathotypes or strains are known exist in one field, one plant, or even one gall. This study was conducted with the objective of testing the prevalence of the coexistence of multiple strains in a single gall. In all, 79 canola clubroot galls were collected from 22 fields across Northern Alberta in 2018. Genomic DNA extracted from these single galls was analyzed using RNase H-dependent PCR (rhPCR). The rhPCR primers were designed to

from a single gall would indicate that it contains two different $P$. brassicae strains. The rhPCR analyses indicated that the $P$. brassicae populations in 50 of the 79 galls consisted of more than one strain. This result emphasizes the need for cautious interpretation of results when a singlegall population is subject to pathotyping or being used as inoculum in plant pathology research. It also confirms that the maintenance of pathotype diversity within single root galls is a common occurrence which has implications for the durability, and stewardship, of single-gene host resistance.
\end{abstract} amplify a partial sequence of a dimorphic gene, with one primer pair specific to one sequence and the other primer pair specific to the alternative sequence. The amplification of both sequences from DNA obtained
Keywords: field crops, oilseeds and legumes, pathogen detection, pathogen diversity, techniques
Clubroot, caused by the protist Plasmodiophora brassicae Woronin, is an important threat to Canadian canola (Brassica napus L.) production (Hwang et al. 2012). Isolates of $P$. brassicae have been classified into pathotypes based on their responses on differential hosts. Commonly used differential host sets include Williams (1966), the European Clubroot Differential set (Buczacki et al. 1975), Somé et al. (1996), and the recently developed Canadian Clubroot Differential set (Strelkov et al. 2018). Historically, P. brassicae pathotypes $2,3,5,6$, and 8 , as defined by the Williams' differentials, were prevalent in Alberta (Strelkov et al. 2006; Xue et al. 2008). In 2013, a new pathotype was detected from one field in Alberta, which was reminiscent of pathotype 5 on the differentials of Williams but was virulent on resistant canola cultivars grown. Because it appeared to be a variant of pathotype 5, this pathotype was referred to as pathotype 5x. Each year since 2013, fields with new virulent pathotypes that can overcome the resistance carried by the canola varieties have been reported (Strelkov et al. 2018). There is no evidence available currently that can support a correlation between the geographic distribution of these new pathotypes and the resistance genes existing in the cultivated canola varieties in the corresponding areas.

With the emergence of new virulent $P$. brassicae pathotypes unable to be controlled by existing clubroot resistance in canola, the ability to rapidly differentiate and identify new pathotypes has

${ }^{\dagger}$ Corresponding authors: M. W. Harding; michael.harding@gov.ab.ca; and J. Feng; jie.feng@gov.ab.ca

Funding: This work was supported by the Western Grains Research Foundation (grant number 2018F092R).

*The $\boldsymbol{e}$-Xtra logo stands for "electronic extra" and indicates that one supplementary table is published online.

The author(s) declare no conflict of interest.

Accepted for publication 27 July 2019.

(C) 2020 The American Phytopathological Society become extremely important for diagnosing resistance breakdown and development of control strategies, as well as breeding for effective resistance. Pathotype differentiation via virulence tests on differential hosts is time consuming and labor intensive. Molecular markers specific to particular pathotypes would be an extremely valuable tool to identify and monitor $P$. brassicae populations because molecular characterizations could provide pathotype designation in days as opposed to months. The currently demonstrated or potential molecular markers for $P$. brassicae pathotyping include a random amplified polymorphic DNA-derived sequence-characterized amplified region marker (Manzanares-Dauleux et al. 2000) specific to P1 as defined by the differentials of Somé et al. (1996), the Cr811 gene (Zhang et al. 2015) specific to pathotype 5 as defined by the differentials of Williams (1966), and a probe-based quantitative PCR marker (Zhou et al. 2018) specific to pathotype 5x. There are also two RNase H-dependent PCR (rhPCR)-based markers (Yang et al. 2018) that can differentiate between the old pathotypes and the new virulent pathotypes present in Alberta, Canada, and five PCRbased markers (Zheng et al. 2018) that can differentiate among pathotypes $4,7,9$, and 11 , as defined by the differentials of Williams (1966). Ideally, any molecular markers should be tested on multiple field isolates to confirm the correlation between the results of these markers and the results obtained from virulence tests on differential hosts before being used as diagnostic tools.

The accuracy of the above-mentioned pathotyping methods may be compromised by the potential heterogeneity of the $P$. brassicae field populations. In fact, the hypothesis that most field galls represent a mixture of pathotypes has been proposed and discussed many times in the clubroot literature over the years. For example, Williams (1966) and Toxopeus et al. (1986) both suggested the presence of heterogeneity in pathogen populations and clubs. This heterogeneity was a driver of efforts to develop methods to isolate single spores of the pathogen (Haji Tinggal and Webster 1981; Jones et al. 1982; Scott 1985; Somé et al. 1996; Xue et al. 2008). However, there has been no molecular method that could be used to identify the mixture of field galls and to verify the purity of single-spore isolates. Recently, Yang et al. (2018) developed an rhPCR method and used this method to test four randomly selected field galls from Alberta. They found that the $P$. brassicae population in each of the four galls 
consisted of at least two strains. This finding emphasized the hypothesis that most field galls in Alberta contain a mixture of $P$. brassicae strains. To verify this hypothesis, 79 single canola galls were collected from 22 Alberta fields in the 2018 growing season. Using the genomic DNA extracted from these single galls as templates, rhPCR was conducted with two primer pairs, each specific to one of the dimorphic sequences of a gene encoding a hypothetical protein. The data obtained provided the first molecular sequence data to support the hypothesis that multiple pathotypes do exist within a single gall, and will help us better understand population dynamics of the clubroot pathogen in Alberta fields and the risks of repeated deployment of the same single resistances for clubroot. These results have implications for $P$. brassicae pathotyping if the starting material is a single gall.

\section{Materials and Methods}

Collection of single galls. Canola plants with clubbed roots were collected from 22 fields in 11 municipalities across Northern Alberta (Fig. 1). A single gall or a part of a single gall, weighting approximately 1 to $2 \mathrm{~g}$, was cut from each fresh plant. These gall samples are termed "single galls" hereafter. In total, 79 single galls were obtained from the 22 fields. The galls were stored in a $-20^{\circ} \mathrm{C}$ freezer until DNA extraction.

DNA extraction. The single galls were ground in liquid nitrogen with a mortar and a pestle. The resultant powder $(100 \mathrm{mg}$ from each single gall) was subjected to genomic DNA extraction. The extraction was performed with the Plant Mini DNA kit (Qiagen Canada, Toronto, ON, Canada) on a QIAcube robotic workstation (Qiagen Canada). DNA extracted from each sample was dissolved in $100 \mu \mathrm{l}$ of sterile deionized water.

Design of primers and gBlocks. One of the two dimorphic genes identified by Yang et al. (2018) was used in this study for primer construction. The two sequences of this gene could be found at the NCBI as partial sequences, with the GenBank accession numbers AM411654 and CDSF01000046, respectively. rhPCR primer pair rhP-3, developed by Yang et al. (2018), was used to specifically amplify the partial AM411654 sequence. A new rhPCR primer pair, rhP-3x, was designed by changing the RNA bases of rhP-3, which enabled rhP-3x to specifically amplify the partial CDSF01000046 sequence. In addition, we also designed another PCR primer by removing the RNA base and the five DNA bases at the $3^{\prime}$ end from the forward rhPCR primers. This PCR primer was named P-Seq and used for sequencing of the rhPCR products. To verify the specificity and efficiency of rhP-3 and rhP-3x, two gBlock gene fragments were designed with sequences corresponding to nucleotides 183 to 554 of AM411654 and nucleotides 267,359 to 267,730 of CDSF01000046, respectively. The two gBlocks were named as G1 and G2, respectively. All primers and the two gBlocks were synthesized by Integrated DNA Technologies in Coralville, IA, U.S.A. (Fig. 2).

rhPCR. DNA samples consisting of different proportions of the gBlocks G1 and G2 were prepared, such that they constituted a set of serial dilutions for both G1 and G2. These DNA samples were used as templates in rhPCR to verify the specificities and efficiencies of rhP-3 and rhP-3x. Each 50- $\mu$ l reaction mixture contained $1 \times$ Promega PCR master mix (Promega Corp., Madison, WI, U.S.A.), RNase H2 (Integrated DNA Technologies) at $0.5 \mathrm{mU} \mathrm{ml}^{-1}, 0.25 \mu \mathrm{M}$ each primer of the primer pair rhP-3 or rhP-3x, and $0.2 \mathrm{pg}$ of template DNA. The rhPCR program consisted of initial denaturation at $94^{\circ} \mathrm{C}$ for $3 \mathrm{~min}$; followed by 30 cycles of denaturation at $94^{\circ} \mathrm{C}$ for $30 \mathrm{~s}$, annealing at $62^{\circ} \mathrm{C}$ for $45 \mathrm{~s}$, and extension at $72^{\circ} \mathrm{C}$ for $1 \mathrm{~min}$; and a final extension at $72^{\circ} \mathrm{C}$ for $5 \mathrm{~min}$. For specific amplification of DNA fragments from the genomic DNA of single clubroot galls, rhPCR was conducted in a $100-\mu 1$ reaction mixture containing $50 \mathrm{ng}$ of template DNA, while the concentration of other ingredients was kept the same as described above. The rhPCR program was also the same as above, except the total number of amplification cycles was changed to 35. All rhPCR were conducted using an Applied Biosystems ProFlex PCR thermal cycler (Fisher Scientific Canada, Ottawa, ON, Canada).

Gel electrophoresis and sequencing. PCR product $(6 \mu \mathrm{l}$ from each reaction) was electrophoresed on $1.5 \%$ agarose gel to check the presence or absence of specific amplicons. The band intensity was quantified using the Bio-Rad Image Lab 5.2.1 software (BioRad Canada, Mississauga, ON, Canada), with the 400-bp band of the 100-bp DNA ladder (Promega Corp.) as the standard. For sequencing, the PCR product was purified from $50 \mu \mathrm{l}$ of each of the selected reactions using the Wizard SV Gel and PCR Cleanup System (Promega Corp.). The purified PCR products were sequenced by the Department of Biological Sciences, University of Alberta (Edmonton, AB, Canada).

\section{Results}

Efficiency of rhP-3 and rhP-3x primers. The rhPCR primer pair rhP-3 amplified a band from the gBlock G1 but not from the gBlock G2. In contrast, rhP-3x amplified a band from G2 but not from G1. Each primer pair could amplify a band from the mixtures of G1 and $\mathrm{G} 2$, even when the percentage of their target sequences was $1 \%$ in the mixture. For each primer pair, the intensity of the band increased along with the proportion of the target gBlock in the mixtures (Fig. 3). These results indicated that the two primer pairs could be used to determine whether a single gall contains two strains, one carrying the target sequence of rhP-3 and the other carrying the target sequence of rhP-3x.

The purity of Alberta field galls. From the DNA of all 79 single galls, either rhP-3 or rhP-3x or both could generate amplicons (Supplementary Table S1). This result confirmed that the sequence polymorphism on this DNA region is prevalent in Alberta's $P$. brassicae populations and exists as a form of dimorphism. The rhP-3 and rhP$3 \mathrm{x}$ primer pairs failed to generate a gel-detectable amplicon from 13 and 16, respectively, of 79 single galls, which resulted in $29(13+16)$ single galls carrying a single sequence form of this dimorphic region. For simplicity, we termed these 29 galls as "pure galls", although we could not conclude whether they contained only one $P$. brassicae strain. On the other hand, we could definitively conclude that the other $50(79-29)$ galls contained a mixture of at least two strains (Table 1). These 50 galls were derived from nine counties and 18

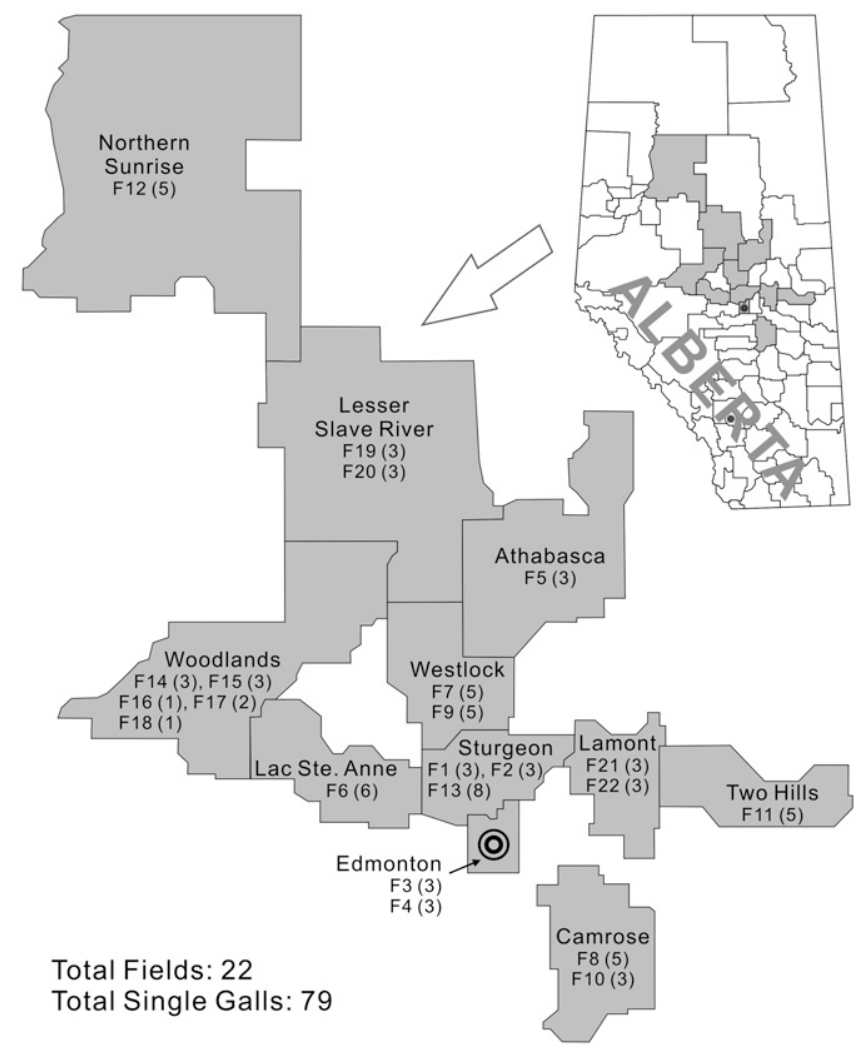

Fig. 1. Geographical origin of clubroot single galls used in this study. County and city names are indicated on the map followed by the field code and number of single galls from each field in parentheses. 
fields (Table 1). In 10 fields (field numbers 3, 4, 5, 6, 9, 10, 13, 14, 15 , and 20), both mixed galls and pure galls coexisted (Table 1).

To confirm the results of rhPCR, the two amplicons generated by rhP-3 and rhP-3x, respectively, from each of nine selected single galls were sequenced (Fig. 4). All of the rhP-3 amplicons had a sequence identical to the corresponding part of the gBlock G1 and all of the rhP-3x amplicons had a sequence identical to the corresponding part of G2. These results confirmed two following conclusions: (i) the generation of both rhP- 3 and rhP-3x amplicons from a single gall is a firm indicator of the gall being a mixture of more than one strain and (ii) the majority of single clubroot galls from Northern Alberta canola fields contain more than one P. brassicae strain.

\section{Discussion}

Based on the sequence data from $13 P$. brassicae populations in Alberta, Yang et al. (2018) found that two genes carried sequence dimorphisms. For each of these two genes, one sequence was specific to the old pathotypes and the alternative sequence was specific to the new, virulent pathotypes. These authors developed two rhPCR primer pairs, with one pair specific to one sequence of one gene and the other pair specific to the alternative sequence of the other gene. By using these primer pairs, the new virulent $P$. brassicae pathotypes could be differentiated from the old pathotypes. Although the usefulness of these primer pairs for $P$. brassicae pathotyping is yet to be determined, because they should be tested on more field populations, they could nevertheless provide concrete evidence to demonstrate that a $P$. brassicae population consists of a mixture of more than one strain. This is because of the advantage of rhPCR being able to specifically amplify the target sequence, even when the target sequence accounted for a very low proportion in a mixture of similar sequences. For example, rhPCR could detect gBlock G1 or G2 even when the targeted template is at $1 \%$ of the mixed DNA of G1 and G2 (Fig. 3). It is impossible to detect such DNAs in such a mixture through sequencing because the majority of DNA sequences could overwhelm the minority in a sequencing reaction. In this study, the two primer pairs rhP-3 and rhP-3x are specific to the dimorphic sequences of a single locus. Generation of amplicons by both primer pairs from one DNA sample would indicate that the template contained both sequences. If the DNA sample was derived from a single clubroot gall, a conclusion can be made that the gall contains at least two strains. This conclusion could further be confirmed by the sequencing data of the two amplicons.
In this study, the rhPCR analyses detected two different strains from 50 of the 79 single galls. However, it is important to note that because only one locus was tested, we could not rule out the possibility that a single gall contained more than two strains. Additionally, this means that the 29 pure galls might also have multiple strains that are not detected using this locus. Previous studies on single-spore isolation indicated that isolates derived from a single gall showed significant variation in virulence (Haji Tinggal and Webster 1981; Jones et al. 1982; Scott 1985; Somé et al. 1996; Xue et al. 2008). Among others, the study from Xue et al. (2008) is more interesting to us because their gall materials came from Alberta. These authors suggested that the population in a single gall might be heterogeneous, and might include a low proportion of spores of physiological races (pathotypes) which were not present in sufficient number to be detected by virulence tests on differential hosts. Furthermore, results from these studies also suggested that single galls can contain more than two strains.

DNA dimorphism seems to be common among $P$. brassicae populations. Yang et al. (2018) sequenced four polymorphic genes in the available strains and found two of them are dimorphic. In human Malaria parasites Plasmodium falciparum (Roy et al. 2008) and $P$. knowlesi (Pinheiro et al. 2015), close relatives of Plasmodiophora

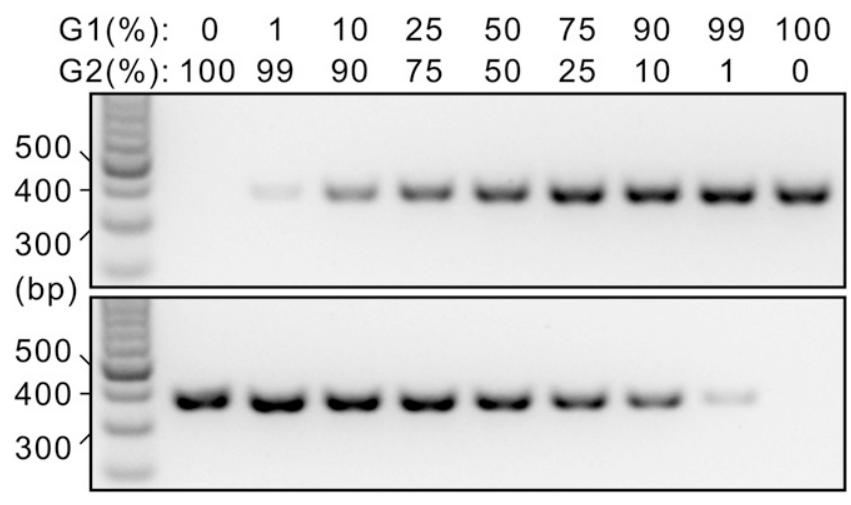

Fig. 3. RNase $\mathrm{H}$-dependent $P C R$ assay against DNA mixtures using the primer pairs rhP-3 (upper panel) and rhP-3x (bottom panel). G1 and G2 = DNA of the gBlock G1 and $\mathrm{G} 2$, respectively. Proportions of the two DNA for each reaction are listed on the top. The leftmost lane is the Promega 100-bp DNA ladder.

G1 CTGGCCAGCATTGACGAAGCGCTGGGTGCCGCTATACCGACCCGGACGTCTCCGCC TCCTGC G2 CTGGCCAGCATTGACGAAGCGCTGGGTGCCGCTATACCAACCCGGACGTCTCCGCC TCCTGC

G1 CGCGAGCTGGGCGAAACCCGACCGTCCAGCAGTCACTCGGTCGCCGAACCAACTGCCAGTGT G2 CGCGAGCTGGGCGAAGCCCGACCGTCCAGCAGTCACTCGGTCACCGAACCAACTCCCAGTGT

G1 GCCATATGACAGACGACGATCTCGACCAGATGCTGCTAGATATCGATGCCGACCTGGACACG G2 GCCATATGACAGACGACGATCTCGACCAGATGTTCTAGATATCGATGCCGACCTGGACACG

G1 GCCGCACCCGACGCCCCGCTGTCTACACCTGGCAAGGGCAACTCAGGGTTTCGGAAGTGCAC G2 GCCGCTCTCGACGCC C CACTGTCTACACCTGTCAAGGGCGATTCAGGGTTTCGGAAGTGCAC

G1 CGTCGTCATGCTAGGTCAGACACCAGCCGCATGCTCCAACCTACGATGTACCATGTGCGACC G2 CGTCGTCATGCTAGGTCAGACACCGGCCGCATGCTCCAACCTACGATGTACCATGTGCGACC G1 ACGTCGTCGTGCGCATTGTCAAACGCGAGTGGGACCCATCCTGCGACTACTTCTTCTTTCGT G2 ACGCCGTCGTGCGCATTTCAAACGCGAATGGACCCATCCTGCGACTACTTCTTCTTTCT

\begin{tabular}{llc}
\hline Primer & \multicolumn{1}{c}{ Forward primer sequence } & Reverse primer sequence \\
\hline rhP-3 & GCTGGGTGCCGCTATACCrGACCCC/3SpC3/ & GTCGCAGGATGGGTCCCArCTCGCC/3SpC3/ \\
rhP-3x & GCTGGGTGCCGCTATACCrAACCCC/3SpC3/ & GTCGCAGGATGGGTCCCArUTCGCC/3SpC3/ \\
P-Seq & GCTGGGTGCCGCTATACC &
\end{tabular}

Fig. 2. Two sequences of the dimorphic DNA region (upper panel) used to design the RNase H-dependent PCR (rhPCR) primers and the sequences of the rhPCR primers (bottom panel). $G 1=$ nucleotides 183 to 554 of the GenBank accession number AM411654. G2 = nucleotides 267,359 to 267,730 of the GenBank accession number CDSF01000046. Nucleotides different between $\mathrm{G} 1$ and $\mathrm{G} 2$ are in bold. Locations of the rhPCR primer pairs are framed. 
brassicae, gene dimorphism has been commonly found. In P. falciparum, the dimorphism has been attributed to a severe population bottleneck when the pathogen transferred from apes to humans by host jump (Roy and Ferreira 2015). Host jumping has been also speculated in $P$. brassicae, although solid evidence supporting this hypothesis is lacking (Neuhauser et al. 2014).

In this study, we concluded that most single galls in Alberta fields contained a mixture of at least two different $P$. brassicae strains. Yang et al. (2018) indicated that the sequence amplified by rhP-3 belongs to the old $P$. brassicae pathotypes and the alternative sequence represented the new virulent pathotypes. If single galls contain different pathotypes, pathotyping by bioassays reliant on virulence testing

Table 1. RNase H-dependent PCR (rhPCR) test on Plasmodiophora brassicae populations derived from 79 single canola galls

\begin{tabular}{|c|c|c|}
\hline $\begin{array}{l}\text { County or city, } \\
\text { field number }\end{array}$ & $\begin{array}{l}\text { Number } \\
\text { of galls }\end{array}$ & $\begin{array}{l}\text { Mixed } \\
\text { galls }\end{array}$ \\
\hline \multicolumn{3}{|l|}{ Athabasca } \\
\hline F5 & 3 & 2 \\
\hline \multicolumn{3}{|l|}{ Camrose } \\
\hline F8 & 5 & 5 \\
\hline F10 & 3 & 1 \\
\hline \multicolumn{3}{|l|}{ Edmonton } \\
\hline F3 & 3 & 2 \\
\hline $\mathrm{F} 4$ & 3 & 2 \\
\hline \multicolumn{3}{|l|}{ Lac Ste. Anne } \\
\hline F6 & 6 & 4 \\
\hline \multicolumn{3}{|l|}{ Lamont } \\
\hline $\mathrm{F} 21$ & 3 & 0 \\
\hline F22 & 3 & 0 \\
\hline \multicolumn{3}{|l|}{ Lesser Slave River } \\
\hline F19 & 3 & 0 \\
\hline $\mathrm{F} 20$ & 3 & 1 \\
\hline \multicolumn{3}{|l|}{ Northern Sunrise } \\
\hline F12 & 5 & 5 \\
\hline \multicolumn{3}{|l|}{ Sturgeon } \\
\hline $\mathrm{F} 1$ & 3 & 3 \\
\hline $\mathrm{F} 2$ & 3 & 3 \\
\hline $\mathrm{F} 13$ & 8 & 7 \\
\hline \multicolumn{3}{|l|}{ Two Hills } \\
\hline F11 & 5 & 0 \\
\hline \multicolumn{3}{|l|}{ Westlock } \\
\hline F7 & 5 & 5 \\
\hline F9 & 5 & 4 \\
\hline \multicolumn{3}{|l|}{ Woodlands } \\
\hline F14 & 3 & 1 \\
\hline F15 & 3 & 1 \\
\hline F16 & 1 & 1 \\
\hline F17 & 2 & 2 \\
\hline F18 & 1 & 1 \\
\hline Total (22 fields) & 79 & 50 \\
\hline
\end{tabular}

a These galls contain more than one $P$. brassicae strain as detected by rhPCR. of single-gall populations on differential hosts may have a significant amount of variability from gall to gall, as indicated in many previous studies (Haji Tinggal and Webster 1981; Jones et al. 1982; Scott 1985; Somé et al. 1996; Xue et al. 2008), making interpretations of results difficult. Similarly, PCR-based molecular markers specific to one pathotype would detect the target only but, currently, cannot characterize the complete range of pathotypes represented in a gall. Amplification of pathotype-specific DNA regions by universal primers followed by amplicon sequencing is a potential strategy for pathotype determination. However, if a single gall contained more than one pathotype, with one pathotype accounting for a very high proportion, the resultant sequencing data would be from this pathotype only (Yang et al. 2018). At this time, the generation of singlespore isolates may need to be a prerequisite for pathotyping of single-gall populations. However, due to the challenges of singlespore isolation in $P$. brassicae, it is impossible to recommend single-spore isolation for all pathotyping work. Until the availability of extensively verified pathotype-specific markers or an easy singlespore isolation protocol, pathotyping of $P$. brassicae will need to continue using root gall populations.

It has not escaped our notice that the prevalence of single galls containing a mixture of $P$. brassicae strains or pathotypes in Alberta fields immediately suggests a possible mechanism for pathotype diversity that is highly responsive to selection when singe-gene host resistance is deployed. We echo the previous suggestion that the rapid rise of new, virulent pathotypes that have overcome the first generation of clubroot resistance in canola is due to the maintenance of multiple pathotypes (Howard et al. 2010; Jones et al. 1982; Strelkov et al. 2016; Tanaka and Ito 2013; Xue et al. 2008). In addition, the prevalence of galls with multiple strains and the fact that they can be easily detected by rhPCR also suggests that the nondominant strains maybe account for higher proportions in the mixture than previously thought. This could be explained by two possible reasons: (i) there is a very short coevolution between strains (or pathotypes) of $P$. brassicae; thus, it is likely that the new pathotypes were introduced to Alberta from other places; or (ii) as a compensation of being soilborne, P. brassicae tends to eliminate intropopulation competition; thus, different strains (or pathotypes) can coexist in the same niche such as a field, a plant, or a single gall. Furthermore, results from this study also suggests that careful stewardship of second-generation clubroot resistance will be critical to avoid selecting for additional nondominant pathotypes that could again cause a rapid loss of resistance.

In conclusion, we found that the rhPCR technique for identification of two clubroot strains has the advantage of detection of the target even when the target is the minority in a mixture. Thus, rhPCR may be a promising system for molecular pathotyping of $P$. brassicae, particularly when a set of dimorphic markers specific to each pathotype is revealed. Studies on more dimorphic genes or DNA regions will help increase the number of such molecular markers that can be used for pathotyping and further resolve the numbers of pathotypes in single root galls. The fact that there are only two sequence forms at the dimorphic region makes genotyping data easy to obtain and interpret. Currently, we are establishing a population of singlespore isolates representing all pathotypes identified in Alberta. With

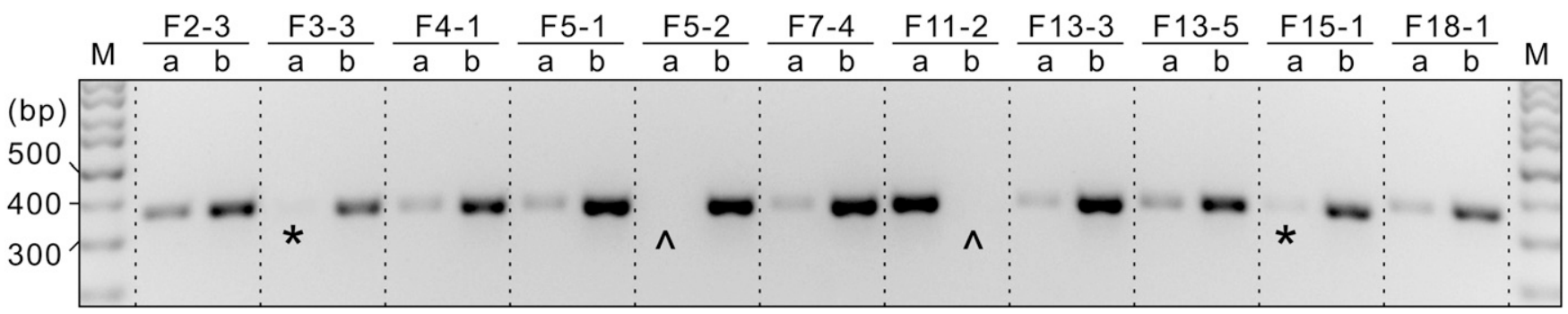

Fig. 4. RNase H-dependent PCR (rhPCR) amplification of the dimorphic sequences from 11 single canola clubroot galls. F2-3 to F18-1 = single galls with the format of "field number-gall number"; $a$ and $b=$ rhPCR primer pairs rhP-3 and rhP3x, respectively; and M = Promega 100-bp DNA ladder. Symbols: * emphasizes weak bands and emphasizes no band. 
the availability of these isolates and more genomic sequence data in the databases, more candidate dimorphic genes will be tested in an attempt to continue to develop an rhPCR system for molecular pathotyping of $P$. brassicae pathotypes.

\section{Literature Cited}

Buczacki, S. T., Toxopeus, H., Mattusch, P., Johnston, T. D., Dixon, G. R., and Hobolth, L. A. 1975. Study of physiologic specialization in Plasmodiophora brassicae: Proposals for attempted rationalization through an international approach. Trans. Br. Mycol. Soc. 65:295-303.

Haji Tinggal, S. H., and Webster, J. 1981. Technique for single spore infection by Plasmodiophora brassicae. Trans. Br. Mycol. Soc. 76:187-190.

Howard, R. J., Strelkov, S. E., and Harding, M. W. 2010. Clubroot of cruciferous crops-New perspectives on an old disease. Can. J. Plant Pathol. 32:43-57.

Hwang, S. F., Strelkov, S. E., Feng, J., Gossen, B. D., and Howard, R. J. 2012. Plasmodiophora brassicae: A review of an emerging pathogen of the Canadian canola (Brassica napus) crop. Mol. Plant Pathol. 13:105-113.

Jones, D. R., Ingram, D. S., and Dixon, G. R. 1982. Characterization of isolates derived from single resting spores of Plasmodiophora brassicae and studies of their interaction. Plant Pathol. 31:239-246.

Manzanares-Dauleux, M. J., Barret, P., and Thomas, G. 2000. Development of a pathotype specific SCAR marker in Plasmodiophora brassicae. Eur. J. Plant Pathol. 106:781-787.

Neuhauser, S., Kirchmair, M., Bulman, S., and Bass, D. 2014. Cross-kingdom host shifts of phytomyxid parasites. BMC Evol. Biol. 14: Article 33.

Pinheiro, M. M., Ahmed, M. A., Millar, S. B., Sanderson, T., Otto, T. D., Lu, W. C., Krishna, S., Rayner, J. C., and Cox-Singh, J. 2015. Plasmodium knowlesi genome sequences from clinical isolates reveal extensive genomic dimorphism. PLoS One 10:e0121303.

Roy, S. W., and Ferreira, M. U. 2015. A new model for the origins of allelic dimorphism in Plasmodium falciparum. Parasitol. Int. 64:229-237.

Roy, S. W., Ferreira, M. U., and Hartl, D. L. 2008. Evolution of allelic dimorphism in malarial surface antigens. Heredity 100:103-110.

Scott, E. S. 1985. Production and characterization of single-spore isolates of Plasmodiophora brassicae. Plant Pathol. 34:287-292.

Somé, A., Manzanares-Dauleux, M. J., Laurens, F., Baron, F., Thomas, G., and Rouxel, F. 1996. Variation for virulence on Brassica napus L. amongst
Plasmodiophora brassicae collections from France derived from single-spore isolates. Plant Pathol. 45:432-439.

Strelkov, S. E., Hwang, S. F., Manolii, V. P., Cao, T., and Feindel, D. 2016. Emergence of new virulence phenotypes of Plasmodiophora brassicae on canola (Brassica napus) in Alberta, Canada. Eur. J. Plant Pathol. 145: 517-529.

Strelkov, S. E., Hwang, S. F., Manolii, V. P., Cao, T., Fredua-Agyeman, R., Harding, M. W., Peng, G., Gossen, B. D., McDonald, M. R., and Feindel, D. 2018. Virulence and pathotype classification of Plasmodiophora brassicae populations collected from clubroot resistant canola (Brassica napus) in Canada. Can. J. Plant Pathol. 40:284-298.

Strelkov, S. E., Tewari, J. P., Smith, E., and Smith-Degenhardt, E. 2006. Characterization of Plasmodiophora brassicae populations from Alberta Canada. Can. J. Plant Pathol. 28:467-474.

Tanaka, S., and Ito, S. I. 2013. Pathogenic and genetic diversity in Plasmodiophora brassicae (clubroot) from Japan. J. Gen. Plant Pathol. 79:297-306.

Toxopeus, H., Dixon, G. R., and Mattusch, P. 1986. Physiological specialization in Plasmodiophora brassicae: An analysis by international experimentation. Trans. Br. Mycol. Soc. 87:279-287.

Williams, P. H. 1966. A system for the determination of races of Plasmodiophora brassicae that infect cabbage and rutabaga. Phytopathology 56:624-626.

Xue, S., Cao, T., Howard, R. J., Hwang, S. F., and Strelkov, S. E. 2008. Isolation and variation in virulence of single-spore isolates of Plasmodiophora brassicae from Canada. Plant Dis. 92:456-462.

Yang, Y., Zuzak, K., Harding, M. W., Strelkov, S., Hwang, S. F., Feindel, D., and Feng, J. 2018. DNA sequence dimorphisms in populations of the clubroot pathogen Plasmodiophora brassicae. Plant Dis. 102:1703-1707.

Zhang, H., Feng, J., Manolii, V. P., Strelkov, S. E., and Hwang, S. F. 2015. Characterization of a gene identified in pathotype 5 of the clubroot pathogen Plasmodiophora brassicae. Phytopathology 105:764-770.

Zheng, J., Wang, X., Li, Q., Yuan, S., Wei, S., Tian, X., Huang, Y., Wang, W., and Yang, H. 2018. Characterization of five molecular markers for pathotype identification of the clubroot pathogen Plasmodiophora brassicae. Phytopathology 108:1486-1492.

Zhou, Q., Hwang, S. F., Strelkov, S. E., Fredua-Agyeman, R., and Manolii, V. P. 2018. A molecular marker for the specific detection of new pathotype 5like strains of Plasmodiophora brassicae in canola. Plant Pathol. 67: 1582-1588. 